\title{
Adherence to anti-retroviral therapy during COVID-19 pandemic among adolescents born HIV-positive
}

\author{
Kudakwashe C. Muchena, Rosemary Kalenga
}

Nelson Mandela University, South Africa

\begin{abstract}
Successful rollout of anti-retroviral therapy (ART) in Africa has resulted in a steep decline in HIV incidence. Even though this has been a long-awaited development, it is not the case for adolescents (10-19 years) born HIV-positive. Given this brief background and dangers imposed by COVID-19 pandemic, adolescents born HIV-positive are at great risk. A systematic review was conducted and reported, according to preferred reporting items for meta-analyses (PRISMA). Studies were drawn from database search performed between April and June 2020 using PsycINFO, Google Scholar, and Sabinet. Results suggested that factors, which negatively impact ART adherence fall into three main levels: 1. Household level: through pressure to share medication; 2. Institutional level: lockdown restrictions due to COVID-19, long distance to facilities, heavy workload due to limited staff, and extended waiting times; 3. Community level: structural barriers, such as HIV stigma and discrimination, gender inequalities, and poor living conditions. Main motivators to remain adherent to ART include having a strong social support system, life goals, and ambitions. This paper highlights the complexity of ART adherence during COVID-19 pandemic. Interventions to improve adherence to ART of adolescents born HIV-positive should address psycho-social factors, such as treatment fatigue, disclosure and family, and household dynamics, in addition to access to ART service delivery during periods of crisis.
\end{abstract}

HIV AIDS Rev 2021; 20, 3: 166-172

DOI: https://doi.org/10.5114/hivar.2021.109249

Key words: HIV, adherence, COVID-19, adolescents.

\section{Introduction}

The COVID-19 pandemic is a public health emergency. As we write, in South Africa, the world counts more than 10 million positive cases and more than 500,000 deaths. The attention that has been given to the COVID-19 pandemic, has resulted in other conditions, such as human immunodeficiency virus (HIV), tuberculosis (TB), malaria, etc. being neglected, yet the diseases contribute to mortalities rate currently recorded. South Africa has so far recorded over 200,000 positive cases and 4,000 deaths due to COVID-19. All deaths cases attributed to the COVID-19 pandemic, in most cases comor-

Address for correspondence: Dr. Kudakwashe Christopher Muchena, Nelson Mandela University, South Africa,

e-mail: s210106646@mandela.ac.za bid (i.e., HIV) have contributed to death rate. Moreover, the risk for HIV as comorbid disease is the leading cause of fatality among COVID-19 death cases, and restrictive measures during the pandemic could also prevent adolescents living with HIV from accessing ART treatment, thereby reducing their adherence [1]. Therefore, ART adherence appears to be a major contributor to fight against COVID-19 fatalities, giving a high number of HIV infections in South Africa.

An analysis of data collected from individuals receiving public sector healthcare services in South Africa has shown that people with HIV are about two-and-a-half times more
Article history:

Received: 16.11.2020

Received in revised form: 01.06.2021

Accepted: 02.06.2021

Available online: 30.09 .2021
International Journal of HIV-Related Problems

HIV \& AIDS $R$ e $v$ i e w 
likely to die of COVID-19 than others [2]. This analysis was the first report on COVID-19 risk in people living with HIV in sub-Saharan Africa. HIV raises the risk of death from COVID-19 in South Africa, with around eight percent of COVID-19 deaths being attributed to HIV [3]. However, the increase in risk is fairly modest, giving high-rate of HIV prevalence in South Africa, relative youth of the population living with HIV show that the absolute number of deaths due to HIV remains small, but warrant attention from researchers.

\section{Adolescents and ART adherence}

Literature suggests that there is a low-rate of adherence to ART among adolescents, leading to high mortality $[4,5]$. Factors, which have been identified as the causes of low adherence include inability to afford transport fares and food, resulting in inability to attend clinic's appointments, malnutrition, and cessation of treatment to avoid increased hunger, which restricts from taking ART. Irregular drug intake was another problem that has been reported a cause for low adherence, and in younger children, this was indicated as a direct result of caregivers not bringing children to clinic or not consistently giving them medicines, whereas in adolescents, it was mainly related to poor adherence to drug regimens. Furthermore, poor adherence to ART among adolescents includes delayed disclosure of HIV status, a desire to conform, and enrolment into boarding school, where it was not possible to supervise intake of medicines from guardians. Even though the access to ART and provision of HIV care has significantly improved in South Africa, a greater proportion of HIV-infected adolescents are likely to survive to their adulthood [6].

In 2007 , it was estimated [7] that $3.4 \%$ of children aged 10 were HIV-infected long-term survivors of mother-tochild transmission (MTCT). They predicted that HIV-related deaths among adolescents' long-term survivors are likely to outnumber those among infants. A reason for this prediction, among other factors, was poor adherence to ART among adolescents. Other factors included lack of services focusing on a specific health needs of HIV-infected adolescents, with its implications for individual and public health, poor adherence during drug resistance, and subsequent treatment failure. This has adverse health consequences for an individual (particularly in settings with limited access to second-line ART); it also carries a risk of HIV transmission. Failure to address emerging sexuality may further increase the risk of unsafe sexual behavior. Delayed disclosure of HIV diagnosis to children, commonly reported by respondents in Sarna's survey [8], was likely to have an additional impact on both drug adherence and secondary HIV prevention.

\section{Theoretical framework}

Adolescents adherence to ART can be viewed from a self-efficacy perspective. Self-efficacy theory says that human motivation influences behaviors and actions, which affects one's life. According to Bandura [9], beliefs in one's capabilities to organize and execute the course of action required to manage prospective situations is the basis of self-efficacy. Therefore, self-efficacy is a belief an individual has that he/ she can accomplish a certain task using their skills under certain circumstances [10]. Lunenburg [11] observed that self-efficacy is a task-specific version of self-esteem. Basic idea behind self-efficacy theory is that performance and motivation are in part determined by how effective people believe they can be [12]. Bandura [13] outlined four sources of information that individuals employ to judge their efficacy, such as performance outcomes (performance accomplishments), vicarious experiences, verbal persuasion, and physiological feedback (emotional arousal). These components help individuals to determine whether they believe they have the capability to accomplish specific tasks. Williams and Williams noted that "individuals with high levels of self-efficacy approach difficult tasks as challenges to master, rather than as threats to be avoided" [14] (p. 455).

\section{Research question and aim of the study}

What challenges are faced by adolescents born HIV-positive receiving primary healthcare in clinics in low socio-economic settings during the COVID-19 pandemic period?

The aim of this study was to provide a detailed analysis of some of the challenges faced by adolescents born HIV-positive in adherence and receiving ART in primary healthcare clinics in low socio-economic settings during the time of COVID-19 pandemic.

\section{Methodology}

A meta-analysis was conducted and reported according to the preferred reporting items for meta-analyses (PRISMA) guidelines [15]. Peer-reviewed articles and grey literature published articles from electronic databases were included in the search.

\section{Search strategy}

Between April and June 2020, a search was performed using PsycINFO, Google Scholar, and Sabinet databases. A total of 1,357 articles were identified in the initial stage of the search, of which, after a screening and removing duplicates, 782 studies remained. Of the 782 articles, 62 were eligible for full text review, and finally, 12 articles were analyzed for the current systematic review.

\section{Results and discussion}

The COVID-19 pandemic has worsened the access to ART drugs and thereby, impacted the adherence of adolescents born HIV-positive who access ART in communities with low social-economic status. This section of the paper discusses some of the major themes that emerged from meta-analysis. 


\section{Stigma and discrimination}

Adolescents' access to care and adherence to ART are mediated by parents and other caregivers. Their uptake of HIV testing and ART, attendance of clinical appointments, and adherence, depend on caregivers' willingness to engage with HIV services, which can be influenced by stigma and discrimination. Fear with which adolescents are stigmatized, reduces caregivers' disclosure [16], with adolescents' ignorance of their status associated with poorer treatment adherence $[17,18]$.

The use of community-based support to promote HIV care engagement has been steadily increasing and often delivered by local community health workers $[19,20]$. These programs can reduce perceived stigma among recipients, including adolescents [21]. The needs of adolescents living with HIV are more sensitive and diverse than those of adults, as they need to simultaneously deal with adult's issues, including disclosure, stigma, and practicing safe sex, while addressing issues traditionally associated with adolescence, such as body image, first sexual experience, peer pressure, and forming of personal identity [22]. These are the issues that are difficult to address during a pandemic.

Psycho-social support (PSS) for HIV-positive children is managed through several organizations, majority of whom provide PSS to vulnerable children more generally rather than to HIV-positive adolescents. The intensity and nature of such provision varies widely. The established guidelines for psycho-social support for HIV-positive children recognize principles for programing combined with performance indicators, but has not yet determined a 'standard of care' for this type of provision $[22,23]$. Therefore, the restriction of movement due to the WHO COVID-19 guidelines makes it difficult for NGOs to support adolescents born HIV-positive, which then affects their adherence to ART regimen.

\section{Restricted access to healthcare services}

Globally, adolescents represent over $40 \%$ of new infections. However, adolescents born HIV-positive are sometime characterized with high-risk sexual behaviors and lack of engagement in healthcare services, which can affect adherence to ART. Despite adherence to ART being critical in controlling viral replication, maintain health and reducing onward viral transmission has limited ART services because of lockdown restrictions imposed by the government to control the spread of COVID-19 [24]. Some service providers have been forced to close due to its' staff members testing COVID-19-positive, thus, restricting the availability of care and support for adolescents born HIV-positive.

Knowing the overall challenges of transition from childhood to adulthood, adolescence are associated with specific challenges (puberty) and vulnerability (early sexual debut, HIV, and STI acquisition). Over and above that, they must cope with clinical and psycho-social impacts of HIV, while carrying a substantial burden of this vulnerable group [25,
26]. HIV-infected adolescents encounter several economic barriers to access HIV care, such as cost of transport and distance to health facility $[27,28]$, and are often unable to address their special health needs, which include disclosure of HIV status, adherence support, stigma and discrimination, sexual and reproductive health, mental healthcare, and legal and social support.

Garvie et al. [29] reported identifying both pill burden and lifestyle issues (i.e., having medications on hand when away from home) as significant barriers to effective adherence. Denial and fear of their HIV infection are common in adolescents, especially in youth, who have recently been diagnosed, which could lead to refusal to initiate or continue ART. Distrust of healthcare workers, misinformation about HIV, and lack of knowledge about the availability and effectiveness of ARV treatment can also be the barriers associated with adolescents care, and their maintenance in care and on successful ART [30].

\section{Institutional level challenges}

Institutional level challenges have been exposed a lot; ART assessment must only be done in local clinics, and they are closed due to COVID-19 among staff members, adolescents born HIV-positive are not transferred to other clinics, which are able to provide their ART. On institutional level, there was no time to plan to solve such challenges of patients that need to access their treatment. As an additional negative effect, adolescents born HIV-positive established a rapport with particular nursing staff who kept their HIV-positivity status undisclosed. Thus, going to another clinic means that other people in the area will know about their HIV-positive status, which becomes a challenge in terms of adherence to ART. Moreover, the access to ART can only be done in local clinics, and home addresses must be provided to make sure that a particular clinic is not over-burdened by people from other areas. Lockdown restrictions due to COVID-19, distance to facilities, heavy workload due to limited staff, and long waiting time have added to challenges of social distancing. Community level and structural barriers include HIV stigma and discrimination, gender inequalities, and poor living conditions. For adolescents born HIV-positive, strong social support system, life goals and ambitions have been reported as motivators to remain adherent. However, when a local clinic closes, adolescents born HIV-positive experience losing a support structure, leaving them with feelings of hopelessness and helplessness.

\section{Interpersonal relationships}

Apart from food insecurity at the household level, which are common in most low socio-economic settings in South Africa, previous studies have identified interpersonal relationships with partners or family members as influencing their decisions to seek testing, engage in care, and stay in care $[31,32]$. The lack of support from family or friends has 
been found to be significant risk factors for non-adherence to ART [33]. Unstable housing and other financial constrains are other important household-level barriers.

At the institutional level, distance to facility (with some services having been closed in compliance with COVID-19 operational guidelines), heavy staff workload, long waiting times, and high fees of usage have been identified as challenges to adherence to ART and retentions in HIV care. At the community level, structural barriers to adherence to ART include HIV-related stigma and discrimination, gender inequalities, weak food systems, political and economic crises, and poor living conditions [34]. Many of these barriers and facilitators to adherence to ART are universally important and relevant in both rich and limited resources communities in South Africa. However, they often manifest differently in various settings. For example, in poor communities resources, poverty itself may impede the ability to obtain treatment through excessive healthcare associated with high costs, while in resources in rich communities, it may correlate to poverty, such as addiction or depression preventing adolescents born HIV-positive from obtaining adequate care or adhering to medications [35].

\section{Social context for ART adherence}

HIV service delivery models to improve adherence outcomes include peer interventions, differentiated service delivery models, and psycho-social support strategies. So far, there have been very few studies on ART adherence among adolescents born with HIV. To support an effective ART adherence services for HIV-positive adolescents, it is necessary to understand the exposure factors that facilitate or prevents the optimal adherence to ART. To date, few studies on ART adherence have used a multi-method approach, despite acknowledged value of combining research methods in behavior and social studies. Any ART adherence support service should provide a comprehensive picture within cultural and structural context of ART in a low socio-economic setting. Literature has demonstrated that improved accessibility to facilities, availability of youth-friendly services, multidisciplinary adolescent HIV clinics, and peer interventions are necessary to enhance adolescents' adherence to ART [36-38]. With a growing programmatic shift toward differentiated HIV service delivery (DSD), evidence on how to implement targeted DSD for adolescents, especially with peer and community interventions, is necessary to recognize during periods of a pandemic. New and optimized strategies to address adolescents living with HIV psycho-social support, mental health, and sexual reproductive health rights needs are urgently required at both individual and program level.

\section{Other challenges of ART adherence}

Low adherence to ARV drugs is commonly encountered in treatment of children and adolescents living with HIV. Other factors, such as medication formulation, frequency of dosing, drug toxicities and their side effects, developmental stage as well as psycho-social, behavioral, and socio-demographic characteristics of adolescents, have been associated with non-adherence to ART. However, no consistent predictors of either good or poor adherence in adolescents have been identified [39-41], thereby posing a threat to adolescents' adherence during crisis, such as the COVID-19 pandemic. Several studies have demonstrated that adherence is not static, and can fluctuate with time on treatment [42].

Literature has demonstrated the difficulty of maintaining high levels of adherence and underscored the need to work with patients and their families to ensure that adherence education, support, and assessment are the integral components of care. Adherence can also be threatened by social and health issues within a family (e.g., substance abuse, poor physical or mental health, unstable housing, poverty, violence, involvement with criminal justice system, and limited social support) $[43,44]$.

It is difficult to ensure adherence during the COVID-19 pandemic, but evaluations should consider social and behavioral factors, which may influence adherence, and should identify individuals needs for intervention. Clinicians should ask patients about their experience with taking medications as well as concerns and expectations about treatment. During this crisis, it is difficult for clinicians to alert patients of potential adverse effects of ARV drugs (e.g., nausea, headaches, abdominal discomfort, sleep disturbances), explain how they can be managed, and emphasize the importance of adherence.

When there are concerns about adherence, the adolescent should be seen and/ or contacted frequently (over the phone, text messaging, email, and social networking, as allowed within the context of legal and regulatory requirements) to assess adherence and determine the need for strategies, which can improve and support ART adherence. Adherence can be optimized using social and community support services.

Patient and caregiver education are essential components of establishing good medication adherence in adolescents. Educating families about adherence should begin before initiating or changing ART medications, and should include a discussion about the goals of therapy, importance of optimizing adherence, and specific plans for supporting and maintaining patient's medication adherence. Caregiver adherence education strategies should include the provision of both information and adherence tools, such as written and visual materials, daily schedule with times and doses of medications, and demonstration of the use of syringes, medication cups, and pillboxes. Additionally, it may be helpful to assess the medication adherence of caregiver or other household members who are currently on ART or other chronic medications.

\section{Improving adolescents' adherence}

The use of behavior modification techniques, especially application of positive reinforcements and use of small incentives (i.e., financial incentives) for taking medications, 
can be effective tools to promote adherence [45]. Treating mental health disorders (e.g., depression) may facilitate adherence to ART [46, 47]. It should be noted, however, that the base of evidence for effective adherence interventions in adolescents and young adults, who are taking daily ART, is limited [48-50].

To improve and support adherence in a pandemic situation, providers should maintain a non-judgmental attitude, establish trust with patients and caregivers, and identify mutually acceptable goals for care. Providers can improve adherence through their relationships with patients' families as well as support comprehensive multidisciplinary care (e.g., with nurses, case managers, pharmacists, social workers, psychiatric care providers), which may also address more complex patients and family needs, including adherence.

In 2015, HIV was the second leading cause of mortality amongst adolescents globally and the leading cause of mortality in sub-Saharan Africa [51]. World Health Organization (WHO) argues that the reason behind this statistic is partly due to insufficient prioritization of adolescent health services in national health programs, poor provision of appropriate HIV testing and counselling (HCT) services as well as sub-standard follow-up care for adolescents, who test HIV-positive and who require ART (WHO, 2013). Further, it is widely reported that even when adolescents do access ART, adherence and retention in care and treatment outcomes are lower compared to adults [52].

Low adherence to ART is one of the most significant challenges to ensure patients achieve and maintain viral load suppression [52]. Factors associated with poor adherence have been categorized as patient-related, structural, provider-related, disease-related, medication-related, and psychological barriers [53]. For adolescents, the transitional life period is characterized by physiological, psychological, and intellectual development, which poses unique challenges to ART adherence [54]. Therefore, the management of adolescents on ART has to consider complexity of biological and psycho-social changes, which take place in their lives as well as its effects on adherence [55-57]. There is a paucity of behavioral research to provide insights into what exactly are the challenges and barriers that adolescents with HIV face when receiving treatment of adult ART program.

\section{Conclusions}

When we first heard about COVID-19 in Wuhan, it seemed too far reach Africa and South Africa in particular. Now, that it is here, and we are experiencing deaths of loved ones and work colleagues dying of COVID-19, we tend to forget about many other diseases that are deadly. Chronic patients are told that because of underlying diseases, they are susceptible to be infected and dying from COVID-19. In all discussions, deadly illnesses, such as HIV and AIDS that require adherence to treatment, are not the focus of health institutions, including local clinics, where most adolescents born with HIV access their treatment. Moreover, health workers are affected by COVID-19 themself, leaving health clinics closed. When all these challenges are unfolding as we watch people dying, we realize that we have not put in place measures to support adolescents born HIV-positive, which struggle not just with physical transformation of their bodies. They also struggle with psychological challenges due to knowing that they are not responsible for being infected with HIV and AIDS, but have to deal with stigma and discrimination associated with being HIV-positive. Furthermore, they face real challenges while seeing their trusted healthcare workers die of COVID-19, or be infected and survived. In addition, due to COVID-19, they are expected study online, which is a challenge when one has spent a good amount of time standing at a clinic hoping to access ARV. All these challenges require psychological support, which is normally not prescribed as a part of treatment to support the expected normal development of adolescents born HIV-positive.

\section{Conflict of interest}

The authors have no conflict of interest.

\section{References}

1. Nyoni T, Okumu M. COVID-19-compliant strategies for supporting treatment adherence among people living with HIV in Sub-Saharan Africa. AIDS Behav 2020. DOI: 10.1007/s10461-020-02888-0 [Epub ahead of print].

2. Alcorn K. People with HIV at greater risk of COVID-19 death in South African study. 2020. Available at: https://www.aidsmap.com/ news/jun-2020/people-hiv-greater-risk-covid-19-death-south -african-study (Accessed: 20.05.2020).

3. Alcorn K. HIV raises the risk of death from COVID-19 in South Africa's Western Cape. 2020. Available at: https://www.aidsmap. com/news/jul-2020/hiv-raises-risk-death-covid-19-south-africas -western-cape (Accessed: 20.05.2020).

4. Bandason T, Langhaug LF, Makamba M, et al. Burden of HIV among primary school children and feasibility of primary schoollinked HIV testing in Harare, Zimbabwe: a mixed methods study. AIDS Care 2013; 25: 1520-1526.

5. Ferrand RA, Munaiwa L, Matsekete J, et al. Undiagnosed HIV infection among adolescents seeking primary health care in Zimbabwe. Clin Infect Dis 2010; 51: 844-851.

6. Judd A, Doerholt K, Tookey PA, et al. Morbidity, mortality, and response to treatment by children in the United Kingdom and Ireland with perinatally acquired HIV infection during 1996-2006: planning for teenage and adult care. Clin Infect Dis 2007; 45: 918-924.

7. Ferrand RA, Corbett EL, Wood R, et al. AIDS among older children and adolescents in Southern Africa: projecting the time course and magnitude of the epidemic. AIDS (London, England) 2009; 23: 2039.

8. Sarna A, Chersich M, Okal J, et al. Changes in sexual risk taking with antiretroviral treatment: influence of context and gender norms in Mombasa, Kenya. Cult Health Sex 2009; 11: 783-797.

9. Bandura A. Comments on the crusade against the causal efficacy of human thought. J Behav Ther Exp Psychiatry 1995; 26: 179-190.

10. Snyder CR, Lopez SJ (eds.). Handbook of Positive Psychology. Oxford University Press; 2001.

11. Lunenburg FC. Self-efficacy in the workplace: implications for motivation and performance. International Journal of Management, Business, and Administration 2011; 14: 1-6.

12. Redmond BF. Self-efficacy theory: do I think that I can succeed in my work? Work attitudes and motivation. The Pennsylvania State University, World Campus; 2010. 
13. Bandura A. Self-efficacy: toward a unifying theory of behavioral change. Psychol Rev 1977; 84: 191.

14. Williams T, Williams K. Self-efficacy and performance in mathematics: reciprocal determinism in 33 nations. Journal of Educational Psychology 2010; 102: 453.

15. Weed M. A potential method for the interpretive synthesis of qualitative research: issues in the development of 'meta-interpretation'. International Journal of Social Research Methodology 2008; 11: 13-28.

16. Krauss BJ, Letteney S, Okoro CN. Why tell children: a synthesis of the global literature on reasons for disclosing or not disclosing an HIV diagnosis to children 12 and under. Front Public Health 2016; 4: 181 .

17. Micheni M, Kombo BK, Secor A, et al. Health provider views on improving antiretroviral therapy adherence among men who have sex with men in coastal Kenya. AIDS Patient Care STDs 2017; 31: 113-121.

18. Nabukeera-Barungi N, Elyanu P, Asire B, et al. Adherence to antiretroviral therapy and retention in care for adolescents living with HIV from 10 districts in Uganda. BMC Infect Dis 2015; 15: 520.

19. Hall LH, Johnson J, Watt I, Tsipa A, O'Connor DB. Healthcare staff wellbeing, burnout, and patient safety: a systematic review. PLoS One 2016; 11: e0159015.

20. Jaffar S, Amuron B, Foster S, et al. Rates of virological failure in patients treated in a home-based versus a facility-based HIV-care model in Jinja, southeast Uganda: a cluster-randomised equivalence trial. Lancet 2009; 374: 2080-2089.

21. Lachman JM, Sherr LT, Cluver L, Ward CL, Hutchings J, Gardner F. Integrating evidence and context to develop a parenting program for low-income families in South Africa. Journal of Child and Family Studies 2016; 25: 2337-2352.

22. Mavhu W, Berwick J, Chirawu P, et al. Enhancing psychosocial support for HIV positive adolescents in Harare, Zimbabwe. PLoS One 2013; 8: e70254.

23. Skovdal M, Magutshwa-Zitha S, Campbell C, Nyamukapa C, Gregson S. Community groups as 'critical enablers' of the HIV response in Zimbabwe. BMC Health Services Research 2013; 13: 195.

24. Jiang H, Zhou Y, Tang W. Maintaining HIV care during the COVID-19 pandemic. Lancet HIV 2020; 7: e308-309.

25. Robson E, Ansell N, Huber US, Gould WT, van Blerk L. Young caregivers in the context of the HIV/AIDS pandemic in sub-Saharan Africa. Population, Space and Place 2006; 12: 93-111.

26. Andrews G, Skinner D, Zuma K. Epidemiology of health and vulnerability among children orphaned and made vulnerable by HIV/ AIDS in sub-Saharan Africa. AIDS Care 2006; 18: 269-276.

27. Geng EH, Bangsberg DR, Musinguzi N, et al. Understanding reasons for and outcomes of patients lost to follow-up in antiretroviral therapy programs in Africa through a sampling-based approach. J Acquir Immune Defic Syndr 2010; 53: 405-411.

28. Amuron B, Namara G, Birungi J, et al. Mortality and loss-to-follo$\mathrm{w}$-up during the pre-treatment period in an antiretroviral therapy programme under normal health service conditions in Uganda. BMC Public Health 2009; 9: 290.

29. Garvie PA, Wilkins ML, Young JC. Medication adherence in adolescents with behaviorally-acquired HIV: evidence for using a multimethod assessment protocol. J Adolesc Health 2010; 47: 504-511.

30. Kay ES, Batey DS, Mugavero MJ. The HIV treatment cascade and care continuum: updates, goals, and recommendations for the future. AIDS Res Ther 2016; 13: 1-7.

31. Geng EH, Bangsberg DR, Musinguzi N, et al. Understanding reasons for and outcomes of patients lost to follow-up in antiretroviral therapy programs in Africa through a sampling-based approach. J Acquir Immune Defic Syndr 2010; 53: 405-411.

32. Amuron B, Namara G, Birungi J, et al. Mortality and loss-to-followup during the pre-treatment period in an antiretroviral therapy programme under normal health service conditions in Uganda. BMC Public Health 2009; 9: 290.
33. Unge C, Södergård B, Marrone G, et al. Long-term adherence to antiretroviral treatment and program drop-out in a high-risk urban setting in sub-Saharan Africa: a prospective cohort study. PLoS One 2010; 5: e13613.

34. Hardon AP, Akurut D, Comoro C, et al. Hunger, waiting time and transport costs: time to confront challenges to ART adherence in Africa. AIDS Care 2007; 19: 658-665.

35. Young S, Wheeler AC, McCoy SI, Weiser SD. A review of the role of food insecurity in adherence to care and treatment among adult and pediatric populations living with HIV and AIDS. AIDS Behav 2014; 18: 505-515.

36. Madiba S, Josiah U. Perceived stigma and fear of unintended disclosure are barriers in medication adherence in adolescents with perinatal HIV in Botswana: a qualitative study. Biomed Res Int 2019; 2019: 9623159.

37. Maskew M, Fox MP, Evans D, et al. Insights into adherence among a cohort of adolescents aged 12-20 years in South Africa: reported barriers to antiretroviral treatment. AIDS Res Treat 2016; 2016: 4161738.

38. Nyoni T, Okumu M. COVID-19-compliant strategies for supporting treatment adherence among people living with HIV in subSaharan Africa. AIDS Behav 2020; 24: 2473-2476.

39. Kolmodin MacDonell K, Jacques-Tiura AJ, Naar S, Fernandez MI, ATN 086/106 Protocol Team. Predictors of self-reported adherence to antiretroviral medication in a multisite study of ethnic and racial minority HIV-positive youth. J Pediatr Psychol 2016; 41: 419-428.

40. Gray ME, Nieburg P, Dillingham R. Pediatric human immunodeficiency virus continuum of care: a concise review of evidence-based practice. Pediatr Clin North Am 2017; 64: 879-891.

41. Schlatter AF, Deathe AR, Vreeman RC. The need for pediatric formulations to treat children with HIV. AIDS Res Treat 2016; 2016: 1654938.

42. Giannattasio A, Albano F, Giacomet V, Guarino A. The changing pattern of adherence to antiretroviral therapy assessed at two time points, 12 months apart, in a cohort of HIV-infected children. Expert Opin Pharmacother 2009; 10: 2773-2778.

43. Cluver L, Meinck F, Toska E, Orkin FM, Hodes R, Sherr L. Multitype violence exposures and adolescent antiretroviral nonadherence in South Africa. AIDS 2018; 32: 975-983.

44. Cluver LD, Hodes RJ, Toska E, et al. 'HIV is like a tsotsi. ARVs are your guns': associations between HIV-disclosure and adherence to antiretroviral treatment among adolescents in South Africa. AIDS 2015; 29 Suppl 1: S57-65.

45. Foster C, McDonald S, Frize G, Ayers S, Fidler S. "Payment by Results" - financial incentives and motivational interviewing, adherence interventions in young adults with perinatally acquired HIV-1 infection: a pilot program. AIDS Patient Care STDs 2014; 28: 28-32.

46. Bucek A, Leu CS, Benson S, et al. Psychiatric disorders, antiretroviral medication adherence, and viremia in a cohort of perinatally HIVinfected adolescents and young adults. Pediatr Infect Dis J 2018; 37: 673-677.

47. Sin NL, DiMatteo MR. Depression treatment enhances adherence to antiretroviral therapy: a meta-analysis. Ann Behav Med 2014; 47: 259-269.

48. Shaw S, Amico KR. Antiretroviral therapy adherence enhancing interventions for adolescents and young adults 13-24 years of age: a review of the evidence base. J Acquir Immune Defic Syndr 2016; 72: 387-399.

49. Judd A, Sohn AH, Collins IJ. Interventions to improve treatment, retention and survival outcomes for adolescents with perinatal HIV-1 transitioning to adult care: moving on up. Curr Opin HIV AIDS 2016; 11: 477-46.

50. Camacho-Gonzalez AF, Chernoff MC, Williams PL, et al. and International Maternal Pediatric Adolescent AIDS Clinical Trials (IMPAACT) P1074 Study Team, 2017. J Pediatric Infect Dis Soc 2017; 6: e22-e29. 
51. UNAIDS. Available at: https://www.unaids.org/sites/default/files/ media_asset/unaids-data-2018_en.pdf 2018

52. Nachega JB, Hislop M, Nguyen H, et al. Antiretroviral therapy adherence, virologic and immunologic outcomes in adolescents compared with adults in southern Africa. J Acquir Immune Defic Syndr 2009; 51: 65-71.

53. Heestermans T, Browne JL, Aitken SC, Vervoort SC, KlipsteinGrobusch K. Determinants of adherence to antiretroviral therapy among HIV-positive adults in sub-Saharan Africa: a systematic review. BMJ Global Health 2016; 1: e000125.

54. Tassiopoulos K, Moscicki AB, Mellins C, Kacanek D, Malee K, Allison S. Pediatric HIV/AIDS Cohort Study. Sexual risk behavior among youth with perinatal HIV infection in the United States: predictors and implications for intervention development. Clin Infect Dis 2013; 56: 283-290.

55. Bekker LG, Johnson L, Wallace M, Hosek S. Building our youth for the future. J Int AIDS Soc 2015; 18: 20027.

56. Williams S, Renju J, Ghilardi L, Wringe A. Scaling a waterfall: a meta-ethnography of adolescent progression through the stages of HIV care in sub-Saharan Africa. J Int AIDS Soc 2017; 20: 21922.

57. Larkan F, Van Wyk B, Stevens P, Saris AJ. Between the clinic and the community: Temporality and patterns of ART adherence in the Western Cape Province, South Africa. African Sociological Review 2015; 19: 26-52 